\title{
Selectivity Profile of the Tyrosine Kinase 2 Inhibitor Deucravacitinib Compared with Janus Kinase 1/2/3 Inhibitors
}

\author{
Anjaneya Chimalakonda $\cdot$ James Burke $\cdot$ Lihong Cheng \\ Ian Catlett · Michael Tagen · Qihong Zhao · Aditya Patel · \\ Jun Shen · Ihab G. Girgis · Subhashis Banerjee $\cdot$ John Throup
}

Received: May 27, 2021 / Accepted: August 18, 2021 / Published online: August 30, 2021

(C) The Author(s) 2021

\begin{abstract}
Introduction: Deucravacitinib, a novel, oral, selective inhibitor of tyrosine kinase 2 (TYK2) signaling, acts via an allosteric mechanism by binding to the enzyme's regulatory domain instead of the catalytic domain. This unique binding provides high functional selectivity for TYK2 versus the closely related Janus kinases (JAKs) $1 / 2 / 3$. Deucravacitinib was efficacious in phase 2 and 3 psoriasis trials, without clinical or laboratory parameters indicative of JAK $1 / 2 / 3$ inhibition being observed. This analysis compared the kinase specificities of deucravacitinib versus JAK $1 / 2 / 3$ inhibitors at therapeutic exposures.
\end{abstract}

Methods: Signaling via JAK $1 / 3$, JAK $2 / 2$, and TYK2/JAK 2 dimers was measured in in vitro whole blood assays. Concentrations providing half-maximal inhibition $\left(\mathrm{IC}_{50}\right)$ in these assays were determined for deucravacitinib and the JAK $1 / 2 / 3$ inhibitors tofacitinib, upadacitinib, and baricitinib. Newly derived whole blood $\mathrm{IC}_{50}$ values were plotted against available pharmacokinetic profiles using doses evaluated in phase $2 / 3$ trials. Simulated average daily inhibition and durations over which concentrations exceeded $\mathrm{IC}_{50}$ were evaluated.

Results: At clinically relevant exposures, projected steady-state deucravacitinib plasma concentrations were higher than TYK2 $\mathrm{IC}_{50}$ for approximately 9-18 h. Maximal plasma concentrations $\left(C_{\max }\right)$ of deucravacitinib were 8 - to

James Burke: employee at the time the analysis was conducted.

A. Chimalakonda $(\bowtie) \cdot J$. Burke $\cdot$ L. Cheng ·

I. Catlett · M. Tagen - Q. Zhao - A. Patel · J. Shen ·

I. G. Girgis · S. Banerjee · J. Throup

Bristol Myers Squibb, Princeton, NJ, USA

e-mail: anjaneya.chimalakonda@bms.com 
17-fold lower than JAK $1 / 3 \quad \mathrm{IC}_{50}$ and $>48$ to $>102$-fold lower than JAK 2/2 IC $_{50}$. Simulated daily average TYK2 inhibition by deucravacitinib ranged from $50 \%$ to $69 \%$. Simulations indicated that tofacitinib, upadacitinib, and baricitinib at steady state exhibited varying degrees of JAK $1 / 3$ (daily average inhibition, 70-94\%) and JAK 2/2 (23\%-67\%) inhibition at therapeutic concentrations, with $C_{\max }$ values 17 - to 33 -fold lower than their TYK2 IC $_{50}$ levels.

Conclusion: At clinically relevant doses and exposures, deucravacitinib demonstrates highly selective inhibition of TYK2 and not JAK 1/2/3. Tofacitinib, upadacitinib, and baricitinib variably inhibit JAK $1 / 2 / 3$ but not TYK 2 . These results indicate that deucravacitinib is a distinct class of kinase inhibitor compared with JAK 1/2/ 3 inhibitors.

\section{PLAIN LANGUAGE SUMMARY}

Psoriasis is a common, chronic inflammatory skin condition that impairs patients' physical health, emotional well-being, work performance, and overall quality of life. Psoriasis and related conditions such as psoriatic arthritis are caused by abnormalities in the immune system. Various drugs are used or explored to treat these conditions, including Janus kinase (JAK) inhibitors; however, JAK inhibitors are associated with a range of side effects such as abnormal changes in blood cell, cholesterol, and triglyceride levels, as well as liver and kidney dysfunction. Deucravacitinib is a new oral drug in development that blocks a key molecule involved in the pathogenesis of psoriasis known as tyrosine kinase 2 (TYK2). This analysis compared the selectivity of deucravacitinib versus approved JAK 1/2/3 inhibitors (tofacitinib, upadacitinib, and baricitinib) for TYK2 and JAK $1 / 2 / 3$ in whole blood assays, using therapeutic doses of each drug. The authors reported that deucravacitinib inhibits TYK2 with minimal or no inhibition of JAK $1 / 2 / 3$. In contrast, tofacitinib, upadacitinib, and baricitinib inhibit JAK 1 , JAK 2, and/or JAK 3 to various degrees but do not inhibit TYK2. These results demonstrate that deucravacitinib is a distinct class of drug compared with the JAK $1 / 2 / 3$ inhibitors. The results of this analysis are consistent with those of two recently completed phase 3 trials in patients with moderate-to-severe plaque psoriasis (POETYK PSO-1 and PSO-2), as well as a phase 2 trial in psoriasis, in which deucravacitinib was efficacious and well tolerated, without clinical or laboratory abnormalities suggestive of JAK 1/2/3 inhibition being observed.

Keywords: Baricitinib; $\quad C_{\max } ; \quad$ Cytokine signaling; Deucravacitinib; $\mathrm{IC}_{50}$; Immunemediated inflammatory disease; Janus kinase signal transducer; Tofacitinib; TYK2; Upadacitinib 


\section{Key Summary Points}

\section{Why carry out this study?}

The Janus kinase (JAK) and signal transducer and activator of transcription signaling pathways play major roles in intracellular cytokine signaling processes involved in the immune system and in the pathogenesis of various immunemediated inflammatory diseases (IMIDs), as well as in non-immune functions such as hematopoiesis and lipid metabolism.

Several active-site-binding inhibitors of JAK $1 / 2 / 3$ are approved or are in development for the treatment of IMIDs. However, these JAK inhibitors are associated with various non-immunerelated adverse effects, including hematologic changes, hyperlipidemia, and renal and hepatic abnormalities, which may be due to the lack of selectivity of these inhibitors for specific family members.

A closely related intracellular kinase, tyrosine kinase 2 (TYK2), can pair with JAK 1 or JAK 2 in specific signaling pathways for cytokines such as type I interferons, interleukin (IL)-23, and IL-12. This analysis compared the functional selectivity of deucravacitinib (a novel, oral, allosteric, selective inhibitor of TYK2) versus approved inhibitors of JAK $1 / 2 / 3$ (tofacitinib, upadacitinib, and baricitinib) for JAK $1 / 2 / 3$ and TYK2 at clinically relevant doses and exposures.

\section{What was learned from this study?}

Simulations indicate that, at clinically relevant doses and exposures, deucravacitinib demonstrates functional inhibition solely of TYK2 and not JAK 1/2/ 3. In contrast, tofacitinib, upadacitinib, and baricitinib inhibit JAK 1, JAK 2, and/ or JAK 3 to varying degrees but do not inhibit TYK2.
These results indicate that deucravacitinib is a distinct class of kinase inhibitor compared with inhibitors of JAK 1/2/3. The high functional selectivity for TYK2 coupled with the lack of inhibition of JAK $1 / 2 / 3$ may contribute to an improved safety profile for deucravacitinib.

\section{INTRODUCTION}

Current oral therapeutic options for immunemediated inflammatory diseases (IMIDs) still do not provide benefit to many patients and may result in adverse effects and long-term toxicity in some patients $[1,2]$. Consequently, novel oral therapies that are safe and efficacious are needed for the management of IMIDs. The Janus kinase signal transducer and activator of transcription (JAK-STAT) signaling pathways mediate intracellular signaling by many mediators [e.g., interleukin (IL)-12, IL-23, and type I interferons (IFNs)] that are involved in the pathogenesis of IMIDs [1-5]. Plaque psoriasis pathogenesis is characterized by chronic inflammation mediated by the innate and adaptive immune systems. Inflammatory cytokines such as IL-23 and tumor necrosis factor-alpha produced by innate immune system cells (e.g., dendritic cells and macrophages) promote the differentiation of T-helper 17 cells; these cells secrete IL-17, tumor necrosis factoralpha, and other inflammatory mediators, which stimulate epidermal cells to produce cytokines and chemokines that attract and activate innate immune system cells. This process results in a positive feedback loop in which IL-23 and IL-17 play central roles in plaque psoriasis pathogenesis [6]. Currently available inhibitors of JAK $1 / 2 / 3$ block cytokine signaling through competitive binding to the ATP-binding sites in the catalytic domains of these enzymes, which are highly conserved and thereby provide poor specificity for the individual kinases $[5,7,8]$. Several JAK inhibitors are either approved or in development for the treatment of IMIDs. Tofacitinib (a JAK 1/2/3 
inhibitor) is approved for the treatment of psoriatic arthritis (PsA), rheumatoid arthritis (RA), and ulcerative colitis; upadacitinib and baricitinib are both approved for treating RA [9-11]. In addition, filgotinib is in development for the treatment of RA, PsA, ulcerative colitis, and Crohn's disease [1]. However, current JAK $1 / 2 / 3$ inhibitors are associated with clinically relevant adverse effects at approved doses with boxed warnings about an increased risk of serious infections, malignancy, and thrombosis [9-11]. Additional adverse effects associated with JAK inhibitors include hematologic changes, hyperlipidemia, gastrointestinal perforation, and hepatic and renal abnormalities $[7,8,12]$. Some of these adverse effects have been attributed to the mechanism of action and/or lack of selectivity of these JAK inhibitors [5].

Tyrosine kinase 2 (TYK2) is also an intracellular signaling tyrosine kinase that plays a central role in the pathophysiology of IMIDs via the regulation of signaling by a limited number of cytokines including IL-12, IL-23, and type I IFNs (IFN- $\alpha$ and IFN- $\beta$ ) $[1,2]$. Deucravacitinib is a novel, oral, selective TYK2 inhibitor with a unique mode of binding to the regulatory (pseudokinase) domain rather than to the highly conserved, ATP-binding site in the catalytic domain [13]. Binding of TYK2 via an allosteric mechanism locks the regulatory domain into an inhibitory conformation with the catalytic domain, thereby trapping TYK2 in an inactive state and preventing receptor-mediated activation and downstream signal transduction [13]. Deucravacitinib exhibited potent binding and inhibition of the TYK2 enzyme in vitro [the concentration providing halfmaximal inhibition $\left(\mathrm{IC}_{50}\right)$ was $0.2 \mathrm{nM}$ in a probe displacement assay] and strongly suppressed IL-12, IL-23, and IFN- $\alpha$ signaling in cellular assays ( $\mathrm{IC}_{50}$ range, $2-19 \mathrm{nM}$ ) [13]. In contrast, deucravacitinib exhibited minimal or no activity against JAK $1 / 2 / 3$ in in vitro kinase binding assays $\left(\mathrm{IC}_{50}>10 \mu \mathrm{M}\right)$ [14], and in cellular signaling assays deucravacitinib showed $>100$-fold greater selectivity for TYK2 versus JAK $1 / 3$ and $>2000$-fold greater selectivity for TYK2 versus JAK $2[13,14]$. The high functional selectivity of deucravacitinib for
TYK2 versus JAK 1/2/3 may result in reduced adverse effects and a differentiated safety profile compared with currently available JAK 1/2/3 inhibitors. Deucravacitinib has been shown to be efficacious in phase 2 and phase 3 trials in plaque psoriasis and had a favorable safety profile, without clinical or laboratory parameters suggestive of inhibition of JAK $1 / 2 / 3$ being observed $[15,16]$.

The aim of the current report is to evaluate the kinase selectivity of deucravacitinib versus the approved JAK 1/2/3 inhibitors tofacitinib, upadacitinib, and baricitinib at their respective therapeutic doses and exposures. Simulations of plasma exposures of these agents in relation to their respective whole blood potency values in relevant assay systems were performed to understand their selectivity versus the individual kinases.

\section{METHODS}

\section{Newly Derived In Vitro Human Whole Blood $\mathrm{IC}_{50}$ Assays}

IL-2-induced signaling is mediated via the JAK 1/JAK 3 heterodimer, resulting in phosphorylation of STAT5 (pSTAT5) [1, 8]. Therefore, modulation of IL-2-induced pSTAT5 was used as a measure of JAK $1 / 3$ pathway inhibition (Table 1). Thrombopoietin (TPO) requires only JAK 2 (JAK 2/JAK 2 homodimer) to mediate phosphorylation of STAT3 proteins. Hence, modulation of TPO-induced pSTAT3 was used as a measure of inhibition of the JAK 2 pathway. IL-12-induced IFN- $\gamma$ production, which signals through the TYK2/JAK 2 heterodimer and is dependent on TYK2 but not JAK 2 activity, was used to measure TYK2 pathway inhibition [13]. All blood donors provided informed consent prior to the collection of any samples used in this study, which was performed in accordance with the Helsinki Declaration of 1964 and its later amendments. The blood collection program was governed by an internal Bristol Myers Squibb committee equivalent to an ethics committee. 
Table 1 In vitro whole blood assays for JAK $1 / 2 / 3$ and TYK2 inhibitors

\begin{tabular}{lll}
\hline $\begin{array}{l}\text { Signaling } \\
\text { kinase }\end{array}$ & Stimulant & Endpoint \\
\hline JAK 1/3 & IL-2 & $\begin{array}{l}\text { STAT5 phosphorylation in T } \\
\text { cells }\end{array}$ \\
JAK 2/2 & TPO & $\begin{array}{c}\text { STAT3 phosphorylation in } \\
\text { platelets }\end{array}$ \\
TYK2/JAK 2 & IL-12 & $\begin{array}{l}\text { IFN- } \gamma \text { production in whole } \\
\text { blood }\end{array}$ \\
\hline
\end{tabular}

$I F N$ interferon, $I L$ interleukin, $J A K$ Janus kinase, STAT signal transducer and activator of transcription, TPO thrombopoietin, TYK2 tyrosine kinase 2

\section{IL-2-Stimulated Phosphorylation of STAT5 in T Cells (JAK 1/3 Activity Measure)}

Human whole blood, collected at $37^{\circ} \mathrm{C}$ using citrate dextrose solution, solution $\mathrm{A}$ as anticoagulant, was incubated with clinically relevant concentrations of deucravacitinib, tofacitinib, upadacitinib, or baricitinib for $30 \mathrm{~min}$ prior to being stimulated with $20 \mathrm{ng} / \mathrm{mL}$ of recombinant human IL-2 (catalog no. PHC0023; Life Technologies Corporation, Carlsbad, CA) for 15 min. Stimulation was terminated by adding BD Phosflow Lyse/Fix Buffer (catalog no. 558049; BD Biosciences, San Jose, CA). Cells were stained with BD Pharmingen fluorescein isothiocyanate mouse anti-human CD3 antibody (catalog no. 555916; BD Biosciences), washed, and permeabilized on ice using BD Phosflow Perm Buffer III (catalog no. 558050; BD Biosciences). Cells were subsequently stained with a BD Phosflow Alexa Fluor 647 mouse STAT5 (pY694) antibody (catalog no. 612599; BD Biosciences) for 30 min prior to flow cytometric analysis (FACS Canto II; BD Biosciences, Erembodegem, Belgium). The amount of phosphorylated STAT5 expression was quantitated by median fluorescence intensity (MFI) after gating on CD3-positive T cells.

\section{TPO-Stimulated Phosphorylation of STAT3 in Platelets (JAK 2 Activity Measure)}

Human whole blood was incubated with deucravacitinib, tofacitinib, upadacitinib, or baricitinib for $30 \mathrm{~min}$, as described previously, prior to being stimulated with $50 \mathrm{ng} / \mathrm{mL}$ of recombinant human TPO (catalog no. 300-18; PeproTech US, Cranbury, NJ) for $15 \mathrm{~min}$. Stimulation was terminated by adding BD Phosflow Lyse/Fix buffer. Cells were stained with a BD Pharmingen fluorescein isothiocyanate mouse anti-human CD61 antibody (catalog no. 555753; BD Biosciences), washed, and permeabilized on ice using BD Phosflow Perm Buffer III. Cells were subsequently stained with BD Phosflow phycoerythrin mouse anti-Stat3 (pY705) antibody (catalog no. 612569; BD Biosciences) for $30 \mathrm{~min}$ prior to flow cytometric analysis. The amount of phosphorylated STAT3 expression was quantitated by MFI after gating on CD61-positive platelets.

\section{IL-12-Stimulated IFN- $\gamma$ Production in Human Blood Cells (TYK2 Activity Measure)}

Human whole blood was incubated with deucravacitinib, tofacitinib, upadacitinib, or baricitinib for $1 \mathrm{~h}$ at $37^{\circ} \mathrm{C}$ prior to being stimulated with $2 \mathrm{ng} / \mathrm{mL}$ of recombinant human IL-12 (catalog no. 200-12; PeproTech US) plus $10 \mathrm{ng} /$ $\mathrm{mL}$ of recombinant human IL-18 (catalog no. 9124-IL-050; R\&D Systems, Inc, Minneapolis, MN) overnight at $37^{\circ} \mathrm{C}$ in a carbon dioxide incubator. Blood was centrifuged at $1200 \mathrm{rpm}$ for $10 \mathrm{~min}$, and plasma was removed for analysis of IFN- $\gamma$ levels using a BD OptEIA human IFN- $\gamma$ enzyme-linked immunosorbent assay kit (catalog no. 555142; BD Biosciences).

The $\mathrm{IC}_{50}$ values, as well as Hill coefficients for inhibition, were determined for deucravacitinib, tofacitinib, upadacitinib, and baricitinib using these assays.

\section{Drug and Dose Selection for Plasma Concentration Profile Simulation}

The exposures of deucravacitinib and JAK $1 / 2 / 3$ inhibitors used in this simulation analysis were derived from phase 2 and phase 3 trials with the respective agents [deucravacitinib $6 \mathrm{mg}$ once daily (QD) and $12 \mathrm{mg}$ QD $[15,16]$, tofacitinib $5 \mathrm{mg}$ twice daily (BID) and $10 \mathrm{mg}$ BID [9], upadacitinib $15 \mathrm{mg}$ QD and $30 \mathrm{mg}$ QD [11], and baricitinib $2 \mathrm{mg}$ QD and $4 \mathrm{mg}$ QD [10]]. 


\section{Deucravacitinib and JAK 1/2/3 Inhibitor PK Profiles and Whole Blood $\mathrm{IC}_{50}$}

Pharmacokinetic (PK) profiles were simulated using parameters derived from published population PK models for tofacitinib, upadacitinib, and baricitinib [17-20] and from internal analyses of deucravacitinib PK parameters from available phase 1 , phase 2 , and phase 3 study data. Briefly, a population PK model was constructed with the reported structural model and parameter values, including population mean parameters (e.g., systemic clearance, volume of distribution, absorption-related parameters) and the intersubject variability for those parameters. Covariate effects were not included in simulating PK profiles.

The PK parameters, including maximum plasma concentration $\left(C_{\max }\right)$, average plasma concentration $\left(C_{\text {ave }}\right)$, and minimum plasma concentration $\left(C_{\text {min }}\right)$, were calculated. Plasma concentrations of deucravacitinib, tofacitinib, upadacitinib, and baricitinib were plotted relative to their whole blood $\mathrm{IC}_{50}$ values. If the whole blood $\mathrm{IC}_{50}$ value was higher than the $C_{\max }$ value, the fold difference between the $\mathrm{IC}_{50}$ and $C_{\max }$ values was calculated. Additionally, key exposure parameters $\left(C_{\max }, C_{\text {ave }}\right.$, and $\left.C_{\min }\right)$ of these agents were plotted relative to their individual whole blood $\mathrm{IC}_{50}$ values.

\section{Daily Average Percent Inhibition by Deucravacitinib and JAK 1/2/3 Inhibitors}

Daily average percent inhibition of TYK2 and JAK $1 / 2 / 3$ signaling was calculated using the following equation based on the average drug concentration, whole blood $\mathrm{IC}_{50}$ value, and the Hill coefficient: daily average percent inhibition $=100 /\left(1+\left[\left(\mathrm{IC}_{50} / X\right)^{\wedge} \mathrm{H}\right]\right)$, where $X$ is the average drug concentration and $\mathrm{H}$ is the Hill coefficient.

Given that these in vitro assays were conducted in whole blood, no adjustments for plasma protein binding were done. Additionally, no further adjustments for cellular penetration by the compounds were considered because the blood-to-plasma concentration ratio of evaluated inhibitors was close to 1.0 (range, 1.16-1.32) [21]. Additionally, no formal statistical comparisons of the daily average percent inhibition were performed because average drug concentration was used in this calculation.

\section{RESULTS}

\section{Deucravacitinib and JAK 1/2/3 Inhibitor In Vitro Whole Blood $\mathrm{IC}_{50}$}

In vitro whole blood $\mathrm{IC}_{50}$ values for deucravacitinib, tofacitinib, upadacitinib, and baricitinib in assays evaluating JAK $1 / 3$, JAK $2 / 2$, and TYK $2 /$

Table 2 Newly derived in vitro whole blood IC $_{50}$ values for deucravacitinib and JAK 1/2/3 inhibitors

\begin{tabular}{|c|c|c|c|c|}
\hline \multirow[t]{2}{*}{ Signaling kinase readout } & \multicolumn{4}{|c|}{ Whole blood $\mathrm{IC}_{50}(95 \% \mathrm{CI}), \mathrm{nM}$} \\
\hline & Tofacitinib & Upadacitinib & Baricitinib & Deucravacitinib \\
\hline $\begin{array}{l}\text { JAK } 1 / 3 \text { (IL-2-induced } \\
\text { pSTAT5) }\end{array}$ & $17(15-19)$ & $8(6.5-9.5)$ & $11(8.7-13)$ & $1646(1446-1872)$ \\
\hline $\begin{array}{l}\text { JAK } 2 / 2 \text { (TPO-induced } \\
\text { pSTAT3) }\end{array}$ & $217(182-258)$ & $41(36-47)$ & $32(28-36)$ & $>10,000(-)$ \\
\hline $\begin{array}{l}\text { TYK2 (IL-12-induced IFN- } \gamma \\
\text { release) }\end{array}$ & $5059(3767-7026)$ & $3685(2346-6208)$ & $2351(1834-2980)$ & $42(29-55)$ \\
\hline
\end{tabular}




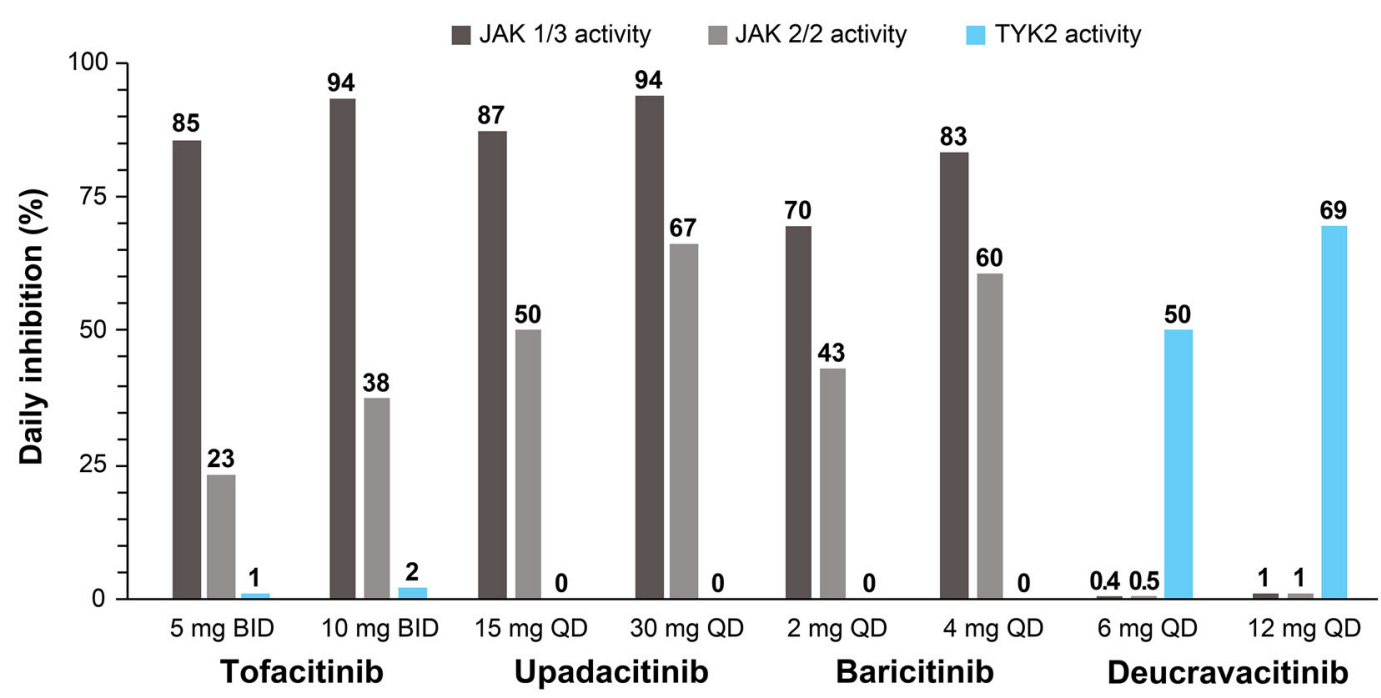

Fig. 1 Simulated daily average percent inhibition by deucravacitinib and JAK $1 / 2 / 3$ inhibitors. JAK $1 / 3$ activity was measured as IL-2-induced STAT5 phosphorylation; JAK 2/2 activity was measured as TPO-induced STAT3 phosphorylation; and TYK2 activity was measured

JAK 2 activity are provided in Table 2. Deucravacitinib was approximately 56- to 120 -fold more potent than tofacitinib, upadacitinib, and baricitinib at inhibiting TYK2-dependent IL12 -induced IFN- $\gamma$ production in human whole blood. In contrast, tofacitinib, upadacitinib, and baricitinib were approximately $97-$ to 206-fold more potent inhibitors of JAK 1/3-dependent IL-2-induced STAT5 phosphorylation in $\mathrm{T}$ cells and at least 46- to 313-fold more potent inhibitors of JAK 2/2-dependent TPOinduced STAT3 phosphorylation in platelets compared with deucravacitinib.

\section{Daily Average Percent Inhibition by Deucravacitinib and JAK 1/2/3 Inhibitors}

At clinically relevant concentrations, simulated daily average percent inhibition (based on in vitro whole blood assay results) of TYK2 by deucravacitinib at therapeutic doses of $6 \mathrm{mg}$ QD and $12 \mathrm{mg}$ QD was $\geq 50 \%$ (Fig. 1). In contrast, simulations indicated minimal effects (daily average percent inhibition of $\leq 1 \%$ ) against IL2-induced STAT5 phosphorylation (JAK 1/3) as IL-12-induced IFN- $\gamma$ production. Numbers above bars represent actual values. $B I D$ twice daily, $I F N$ interferon, $I L$ interleukin, $J A K$ Janus kinase, $Q D$ once daily, $S T A T$ signal transducer and activator of transcription, TPO thrombopoietin, TYK2 tyrosine kinase 2

and TPO-induced STAT3 phosphorylation (JAK $2 / 2$ ) by deucravacitinib. Simulations indicated that tofacitinib, upadacitinib, and baricitinib plasma concentrations exhibited varying degrees of daily average inhibition against IL2-induced STAT5 phosphorylation (JAK 1/3 activity; daily average inhibition, 70-94\%) and TPO-induced STAT3 phosphorylation (JAK 2/2 activity; daily average inhibition, 23-67\%), and no meaningful inhibition against IL-12-induced IFN- $\gamma$ production (TYK2 activity; daily average inhibition, $\leq 2 \%$ ).

\section{Deucravacitinib and JAK 1/2/3 Inhibitor PK Profiles and Whole Blood $\mathrm{IC}_{50}$ Values}

At therapeutic doses of $6 \mathrm{mg}$ QD and $12 \mathrm{mg}$ QD, deucravacitinib plasma concentrations were higher than the TYK2 whole blood $\mathrm{IC}_{50}$ value for approximately 9 and $18 \mathrm{~h}$, respectively, of the daily dosing interval and were substantially lower than the JAK $1 / 3$ and JAK $2 / 2$ IC $_{50}$ values throughout the dosing interval (Figs. 2 and 3). Deucravacitinib $\mathrm{C}_{\max }$ levels at these doses remained approximately 8- to 17-fold lower than the JAK $1 / 3 \mathrm{IC}_{50}$ and $>48$ - to $>102$-fold 


\section{Tofacitinib}

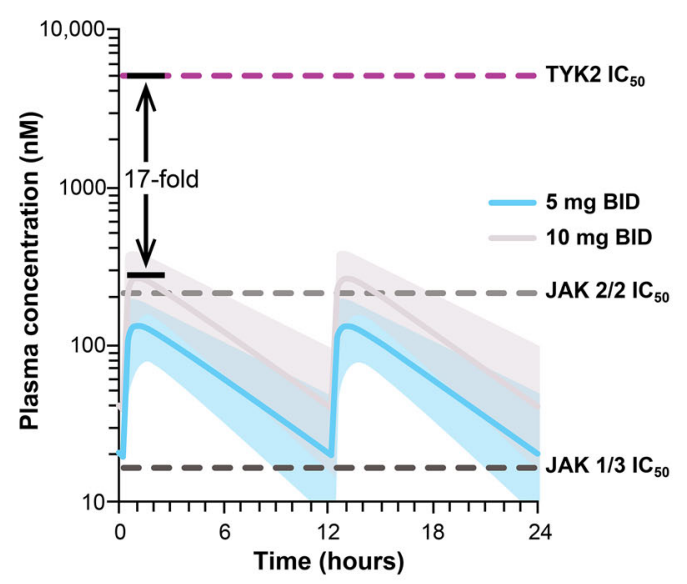

Baricitinib

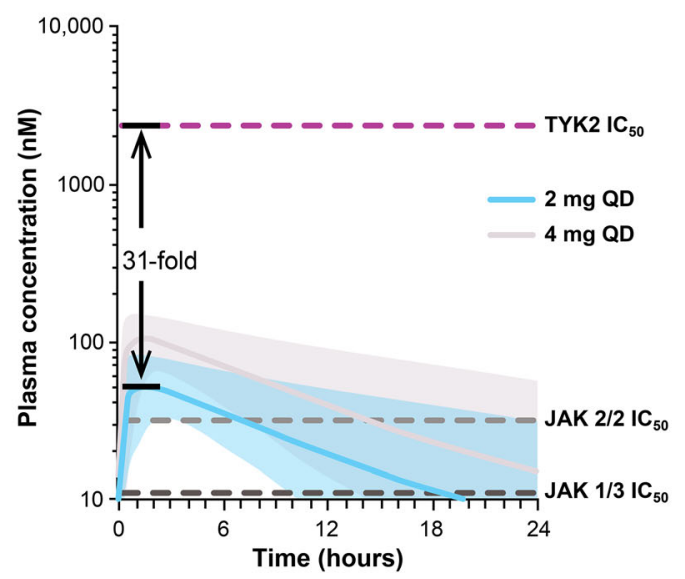

Fig. 2 Deucravacitinib and JAK 1/2/3 inhibitor plasma concentrations over time and whole blood $\mathrm{IC}_{50}$ values. Variability in plasma concentration profiles, represented by $90 \%$ prediction intervals, is indicated by the shaded regions. Arrows and fold-increase values pertain to the

lower than the JAK $2 / 2 \mathrm{IC}_{50}$ (Fig. 2). The $C_{\max }$ $C_{\text {ave, }}$ and $C_{\min }$ values were all higher than or close to the TYK2 $\mathrm{IC}_{50}$ value, but were lower than the JAK $1 / 3$ and JAK $2 / 2 \quad \mathrm{IC}_{50}$ values for deucravacitinib (Fig. 4).

In contrast, tofacitinib, upadacitinib, and baricitinib plasma concentrations at their approved doses were higher than their individual JAK $1 / 3$ IC $_{50}$ values over most of the dosing interval but were 17- to 33-fold lower than their TYK2 IC $_{50}$ values (Fig. 2). Additionally, upadacitinib and baricitinib plasma concentrations exceeded their respective JAK $2 / 2 \quad \mathrm{IC}_{50}$ values
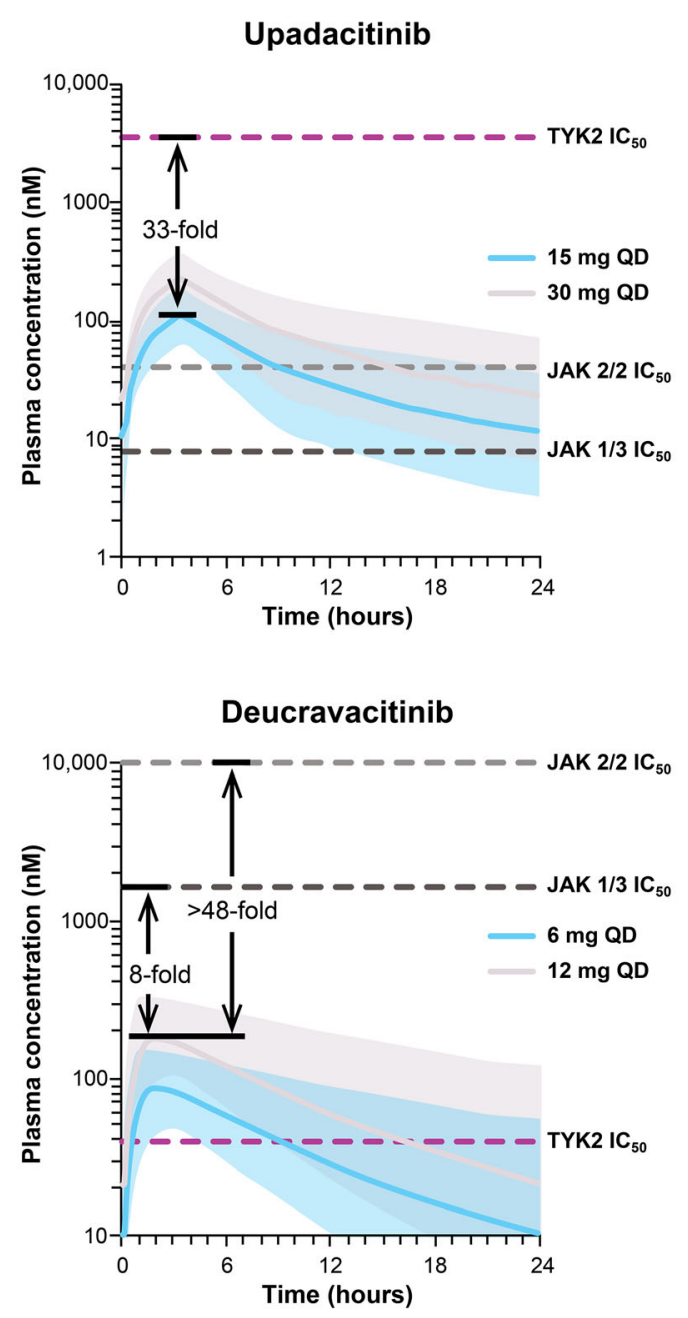

highest approved dose for each agent. Tofacitinib, upadacitinib, and baricitinib: margins to TYK2 $\mathrm{IC}_{50}$ are provided for the highest approved dose. $B I D$ twice daily; $I C_{50}$ halfmaximal inhibitory concentration; $J A K$ Janus kinase, $Q D$ once daily, TYK2 tyrosine kinase 2

over part of the dosing interval, especially at higher doses. The $C_{\max }, C_{\mathrm{ave}}$, and $C_{\min }$ values for tofacitinib, upadacitinib, and baricitinib were all 16- to 335 -fold lower than TYK2 IC $_{50}$ values, but were above or within range of JAK $1 / 3$ and JAK $2 / 2$ IC $_{50}$ values (Fig. 4).

\section{DISCUSSION}

Previous studies have established that many JAK $1 / 2 / 3$ inhibitors are associated with various adverse effects at therapeutic doses, including 


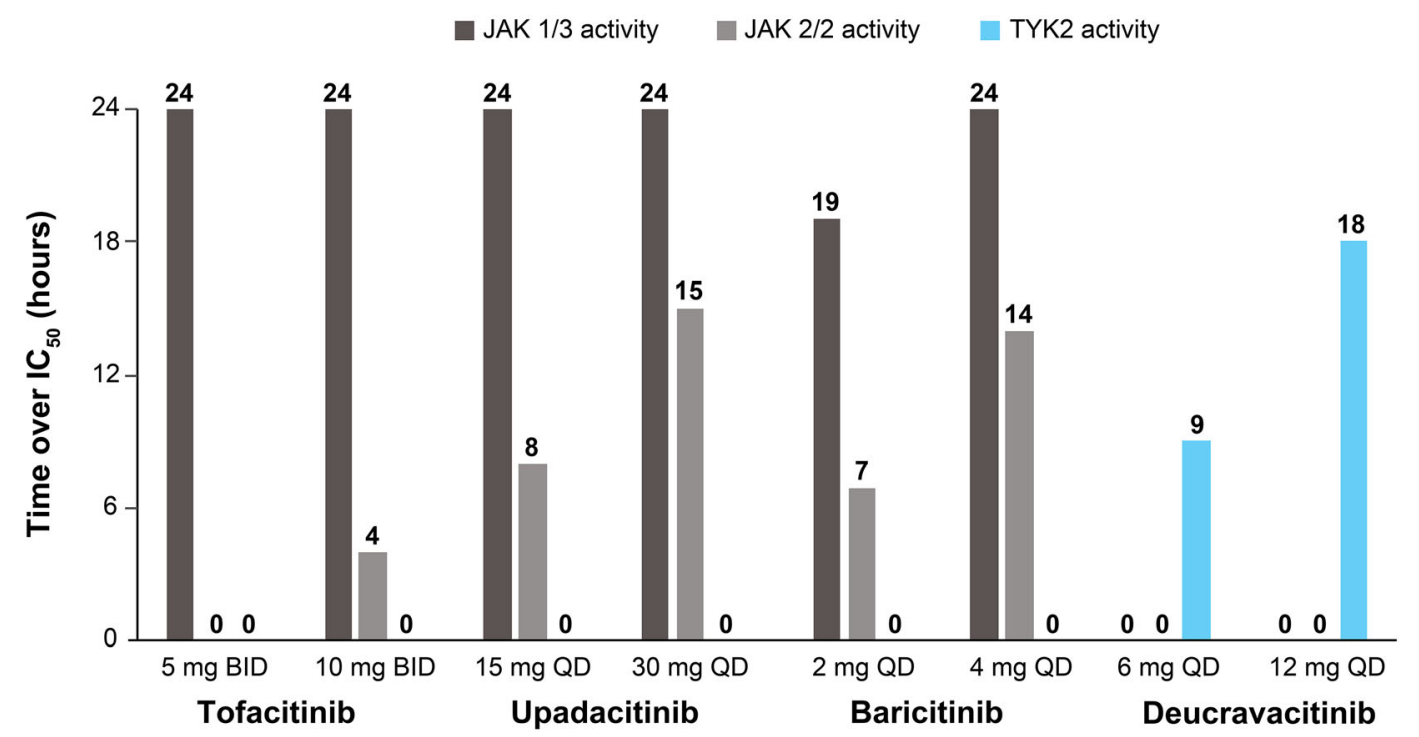

Fig. 3 Deucravacitinib and JAK $1 / 2 / 3$ inhibitor plasma concentration time above $\mathrm{IC}_{50}$. Numbers above bars represent actual values. $B I D$ twice daily, $I C_{50}$ half-maximal

hematologic changes (decreased hemoglobin levels; decreased lymphocyte, natural killer cell, neutrophil, and platelet counts; increased risk of pulmonary embolism and venous thrombosis), hyperlipidemia (elevated high-density lipoprotein cholesterol, low-density lipoprotein cholesterol, and triglyceride levels), and renal (increased serum creatinine levels) and hepatic (increased liver enzyme levels) abnormalities $[7,8,12]$. Although the etiology of some of these adverse effects has not been determined, available evidence suggests that they may arise due to inhibition of select JAK $1 / 2 / 3$ pathways. For example, hyperlipidemia, neutropenia, and liver enzyme elevations are postulated to be due to inhibition of JAK 1/2-mediated IL-6 signaling, considering these effects are also seen with IL-6 antagonists [7, 22]. Hematologic abnormalities can occur due to inhibition of JAK 2-mediated signaling by hematopoietic growth factors (erythropoietin, granulocyte-macrophage colony-stimulation factor, and TPO) [7, 22]. Notably, as demonstrated in the current analysis, lack of inhibition of TYK2 by tofacitinib, upadacitinib, and baricitinib at therapeutic concentrations suggests that the above adverse effects associated with these JAK inhibitory concentration, $J A K$ Janus kinase, $Q D$ once daily, $T Y K 2$ tyrosine kinase 2

$1 / 2 / 3$ inhibitors are unlikely to be related to TYK2 inhibition.

The results from the current analyses confirm that deucravacitinib is a highly selective TYK2 inhibitor with minimal or no activity against JAK $1 / 2 / 3$ at clinically relevant doses and concentrations. Deucravacitinib plasma concentrations were higher than the TYK2 whole blood $\mathrm{IC}_{50}$ value over many hours after dosing. In contrast, plasma concentrations of deucravacitinib were substantially lower than its JAK $1 / 2 / 3$ whole blood IC $_{50}$ values.

As noted earlier, deucravacitinib was efficacious in a phase 2 clinical trial in patients with moderate-to-severe plaque psoriasis. Deucravacitinib was associated with low rates of treatment discontinuation due to adverse events (2-7\% versus $4 \%$ for placebo) in that trial [15]. Selective TYK2 inhibition is expected to reduce the potential for treatment-related adverse effects generally associated with JAK $1 / 2 / 3$ inhibitors [15]. No significant changes were observed in hematologic parameters (lymphocytes, natural killer cells, neutrophils, platelets, erythrocytes, and leukocytes) or in serum levels of lipids (total cholesterol, highdensity lipoprotein cholesterol, and triglycerides), creatinine, or liver enzymes with 

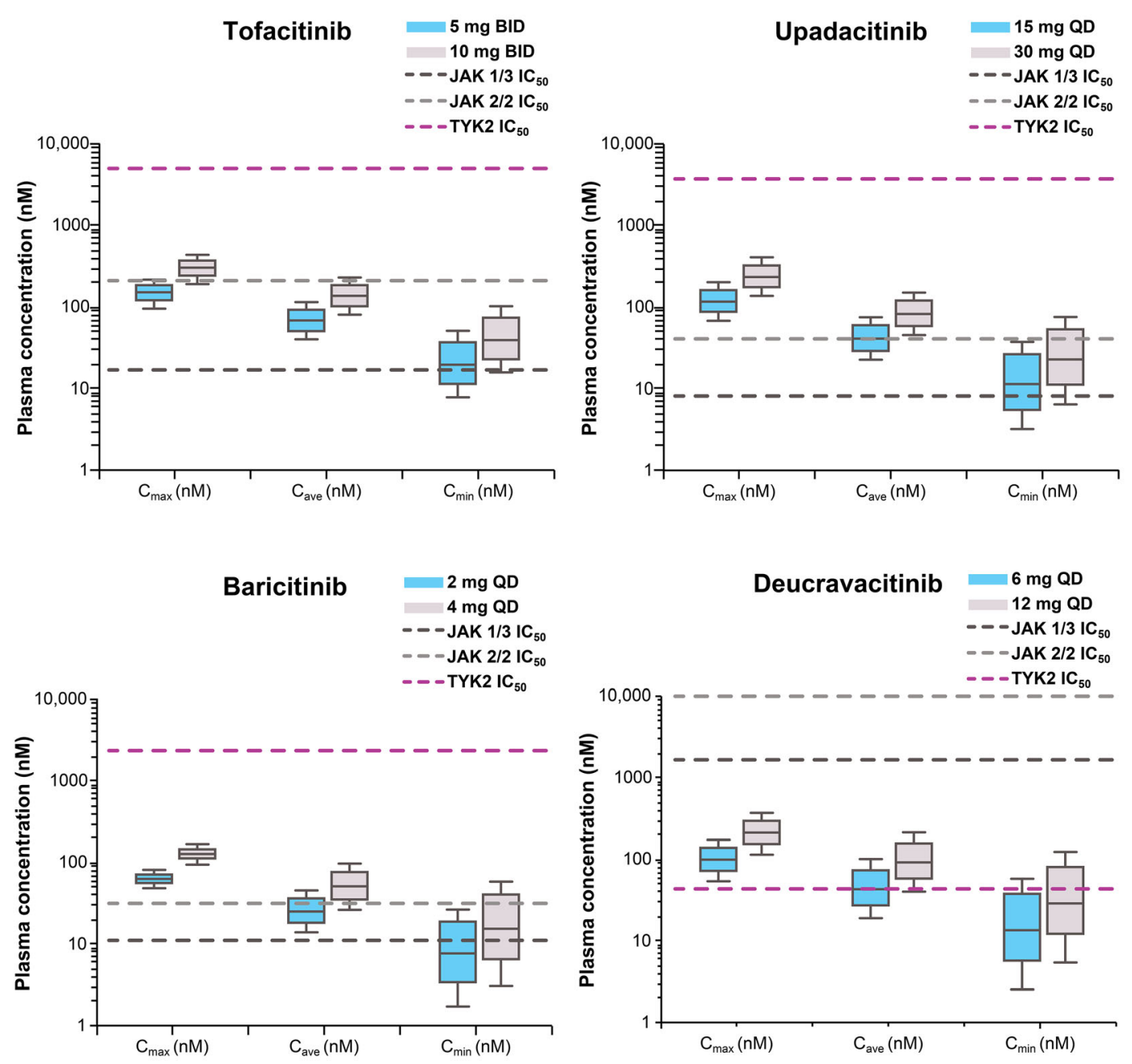

Fig. 4 Deucravacitinib and JAK $1 / 2 / 3$ inhibitors' pharmacokinetic parameters and whole blood $\mathrm{IC}_{50}$ values. $B I D$ twice daily, $C_{\text {ave }}$ average plasma concentration, $C_{\max }$ maximum plasma concentration, $C_{\text {min }}$ minimum or trough

plasma concentration, $I C_{50}$ half-maximal inhibitory concentration, JAK Janus kinase, $Q D$ once daily, TYK2 tyrosine kinase 2

therapeutic doses of deucravacitinib [15, 23]. Two recently completed phase 3 trials [NCT03624127 (POETYK PSO-1) and NCT03611751 (POETYK PSO-2)] demonstrated that deucravacitinib was more efficacious than placebo and apremilast, and was well tolerated in patients with moderate-to-severe plaque psoriasis [16]. As in the phase 2 trial, no clinically meaningful changes from baseline were observed in hematologic, lipid, or other laboratory parameters, confirming that selective TYK2 inhibition by deucravacitinib reduces the incidence of adverse events characteristic of JAK

1/2/3 inhibitors [16]. Additional phase 3 trials are also evaluating the efficacy and safety of deucravacitinib in patients with moderate-tosevere plaque psoriasis [NCT04167462 (POETYK PSO-3), NCT03924427 (POETYK PSO-4), and NCT04036435 (POETYK PSO-LTE)]. The efficacy and safety of deucravacitinib have been or are being evaluated in phase 2 trials in active PsA (NCT03881059), moderate-to-severe inflammatory bowel disease (ulcerative colitis: LATTICEUC, NCT03934216; Crohn's disease: LATTICECD, NCT03599622), and lupus (PAISLEY SLE, NCT03252587; PAISLEY LN, NCT03943147; 
and a long-term safety and efficacy trial, NCT03920267). Results of the phase 2 trial in active PsA demonstrated that deucravacitinib was more efficacious than placebo over a treatment period of 16 weeks. Treatment was well tolerated, and no unexpected safety issues were identified [24].

In the current study, the JAK $1 / 2 / 3$ inhibitors, tofacitinib, upadacitinib, and baricitinib, did not inhibit TYK2 at clinically meaningful doses and exposures and exhibited varying degrees of JAK $1 / 2 / 3$ inhibition. These findings are consistent with other published literature reports, which indicate a lack of meaningful differences in JAK inhibition profiles among these inhibitors [21, 25-27]. A comparison of tofacitinib-, upadacitinib-, and baricitinib-mediated regulation of cytokine signaling in human leukocytes revealed that each JAK inhibitor modulated distinct cytokine pathways to varying degrees, and none of these inhibitors potently or continuously inhibited a specific cytokine signaling pathway throughout the dosing interval [25]. In a previous study, average daily inhibition of JAK 1/3-related and JAK 2-related endpoints in cellular assays for tofacitinib, upadacitinib, and baricitinib were comparable with values reported in this analysis [25]. Dowty et al. used an integrated modeling approach and calculated cytokine receptor inhibition profiles for tofacitinib, upadacitinib, and baricitinib [21]. Although the absolute JAK $1 / 3$, JAK 2, and TYK2 inhibition related measures differed from values reported in the current study, Dowty et al. also reported that these three JAK inhibitors have generally similar cytokine receptor inhibition profiles at clinically efficacious doses [21]. Similar to the current study, Dowty et al. evaluated inhibition of the IL-12-mediated TYK2/JAK 2 pathway [21]. However, STAT phosphorylation (pSTAT4) was used as an endpoint in the Dowty et al. study, whereas we utilized a relatively downstream functional readout (IFN- $\gamma$ production). Therefore, the absolute inhibition $\left(\mathrm{IC}_{50}\right)$ values from these two assays may not be directly comparable. It should also be noted that $\mathrm{IC}_{50}$ values of individual drugs may vary among laboratories (likely due to varying assay conditions, personnel, and systems). Therefore, assessments that compare all compounds within the same laboratory facilitate more accurate intercompound comparisons.

One of the key strengths of this analysis is that this is the first report describing the selectivity of the TYK2 inhibitor deucravacitinib, considering that previous analyses had focused only on JAK $1 / 2 / 3$ inhibitors [21, 25-27]. In addition, the selectivity of deucravacitinib and the JAK $1 / 2 / 3$ inhibitors was compared using the same whole blood $\mathrm{IC}_{50}$ assay conditions, which facilitate more accurate intercompound comparison. Several limitations need to be considered when interpreting results presented here. This analysis was restricted to three stimuli in whole blood assays (Table 1) as surrogates of TYK2 and JAK 1/2/3 activity but did not explore other functional measures of TYK2 and JAK $1 / 2 / 3$ inhibition. Only one assay provided a cytokine readout, whereas the others measured intracellular STAT phosphorylation. However, numerous assays evaluated in a prior deucravacitinib publication demonstrated specific inhibition of TYK2 pathways by deucravacitinib compared with multiple JAK $1 / 2 / 3$ inhibitors [13]. Another limitation is that the impact of pathway modulation on downstream measures such as relevant changes in JAK 1/2/3-related biomarkers after administration to patients cannot be determined using this analysis. Future analyses using assays evaluating downstream biomarkers of JAK $1 / 2 / 3$ inhibition in clinical trials may provide additional information in this regard. However, available data from the phase 2 and phase 3 trials in plaque psoriasis indicated no meaningful changes in postulated JAK 1/3 and JAK 2 biomarkers such as serum lipids, neutrophils, and hemoglobin following treatment with deucravacitinib $[15,23]$. Therefore, along with available data from the phase 2 and phase 3 trials, meaningful deucravacitinib-induced changes in JAK 1/2/3related biomarkers are not anticipated after administration to patients $[15,23]$.

\section{CONCLUSION}

This integrated analysis and the simulations indicate that deucravacitinib has high 
functional selectivity for TYK2 and does not inhibit JAK $1 / 2 / 3$ at clinically relevant doses and exposures. In contrast, tofacitinib, upadacitinib, and baricitinib inhibit JAK $1 / 2 / 3$ to varying degrees but do not inhibit TYK2 at therapeutic concentrations. These results confirm that deucravacitinib is a distinct class of kinase inhibitor compared with JAK $1 / 2 / 3$ inhibitors. The high functional selectivity for TYK2 versus JAK $1 / 2 / 3$ may contribute to the favorable benefit-risk profile of deucravacitinib in patients.

\section{ACKNOWLEDGEMENTS}

The authors thank the patients who donated blood samples for this analysis. The authors also thank Joann Strnad for her assistance in assay performance and development of relevant study reports.

Funding. This analysis was sponsored by Bristol Myers Squibb, Princeton, NJ, USA. Bristol Myers Squibb also funded Dermatology and Therapy's Rapid Service Fees.

Medical Writing and/or Editorial Assistance. Professional medical writing from Ann Marie Fitzmaurice, $\mathrm{PhD}$ and editorial assistance were provided by Peloton Advantage, LLC, an OPEN Health company, Parsippany, NJ, USA, and were funded by Bristol Myers Squibb.

Authorship. All named authors meet the International Committee of Medical Journal Editors (ICMJE) criteria for authorship for this article, take responsibility for the integrity of the work as a whole, and have given their approval for this version to be published.

Author Contributions. AC contributed to the conception and study design; JB, LC, QZ, and JS contributed to data acquisition; AC, JB, IC, IGG, MT, and SB contributed to data analysis; and AC, JB, IC, QZ, AP, IGG, SB, and JT contributed to data interpretation. All authors reviewed this manuscript and gave final approval for publication.
Disclosures. Anjaneya Chimalakonda, Lihong Cheng, Ian Catlett, Michael Tagen, Qihong Zhao, Aditya Patel, Jun Shen, Ihab G. Girgis, Subhashis Banerjee, and John Throup are employees of and shareholders in Bristol Myers Squibb; James Burke was an employee and shareholder of Bristol Myers Squibb at the time the analysis was conducted.

Compliance with Ethics Guidelines. This study was performed in accordance with the Helsinki Declaration of 1964 and its later amendments. All blood donors provided informed consent prior to the collection of any samples used in this study. The blood collection program was governed by an internal Bristol Myers Squibb committee equivalent to an ethics committee.

Data Availability. Bristol Myers Squibb's policy on data sharing is located at https:// www.bms.com/researchers-and-partners/ independent-research/data-sharing-requestprocess.html.

Open Access. This article is licensed under a Creative Commons Attribution-NonCommercial 4.0 International License, which permits any non-commercial use, sharing, adaptation, distribution and reproduction in any medium or format, as long as you give appropriate credit to the original author(s) and the source, provide a link to the Creative Commons licence, and indicate if changes were made. The images or other third party material in this article are included in the article's Creative Commons licence, unless indicated otherwise in a credit line to the material. If material is not included in the article's Creative Commons licence and your intended use is not permitted by statutory regulation or exceeds the permitted use, you will need to obtain permission directly from the copyright holder. To view a copy of this licence, visit http://creativecommons.org/licenses/by$\mathrm{nc} / 4.0 /$. 


\section{REFERENCES}

1. Nogueira M, Puig L, Torres T. JAK inhibitors for treatment of psoriasis: focus on selective TYK2 inhibitors. Drugs. 2020;80(4):341-51.

2. Kvist-Hansen A, Hansen PR, Skov L. Systemic treatment of psoriasis with JAK inhibitors: a review. Dermatol Ther (Heidelb). 2020;10(1):29-42.

3. Balogh EA, Bashyam AM, Ghamrawi RI, Feldman SR. Emerging systemic drugs in the treatment of plaque psoriasis. Expert Opin Emerg Drugs. 2020;25(2):89-100.

4. Howell MD, Kuo FI, Smith PA. Targeting the Janus kinase family in autoimmune skin diseases. Front Immunol. 2019;10:2342.

5. Baker KF, Isaacs JD. Novel therapies for immunemediated inflammatory diseases: what can we learn from their use in rheumatoid arthritis, spondyloarthritis, systemic lupus erythematosus, psoriasis, Crohn's disease and ulcerative colitis? Ann Rheum Dis. 2018;77(2):175-87.

6. Schön MP, Erpenbeck L. The interleukin-23/interleukin-17 axis links adaptive and innate immunity in psoriasis. Front Immunol. 2018;9:1323.

7. Gadina M, Chisolm DA, Philips RL, et al. Translating JAKs to jakinibs. J Immunol. 2020;204(8): 2011-20.

8. Winthrop KL. The emerging safety profile of JAK inhibitors in rheumatic disease. Nat Rev Rheumatol. 2017;13(4):234-43.

9. Xeljanz (tofacitinib) [package insert]. New York: Pfizer Inc.; 2020. Available at: http://labeling.pfizer. com/ShowLabeling.aspx?id=959. Accessed 23 Aug 2021.

10. Olumiant (baricitinib) [package insert]. Indianapolis, IN: Lilly USA, LLC; 2020. Available at: http://pi.lilly.com/us/olumiant-uspi.pdf. Accessed 23 Aug 2021.

11. Rinvoq (upadacitinib) [package insert]. Sligo, Ireland: AbbVie Ireland NL B.V.; 2020. Available at: https://www.rxabbvie.com/pdf/rinvoq_pi.pdf. Accessed 23 Aug 2021.

12. Olivera PA, Lasa JS, Bonovas S, et al. Safety of Janus kinase inhibitors in patients with inflammatory bowel diseases or other immune-mediated diseases: a systematic review and meta-analysis. Gastroenterology. 2020;158(6):1554-73.e12.

13. Burke JR, Cheng L, Gillooly KM, et al. Autoimmune pathways in mice and humans are blocked by pharmacological stabilization of the TYK2 pseudokinase domain. Sci Transl Med. 2019;11(502): $1-16$.

14. Wrobleski ST, Moslin R, Lin S, et al. Highly selective inhibition of tyrosine kinase 2 (TYK2) for the treatment of autoimmune diseases: discovery of the allosteric inhibitor BMS-986165. J Med Chem. 2019;62(20):8973-95.

15. Papp K, Gordon K, Thaci D, et al. Phase 2 trial of selective tyrosine kinase 2 inhibition in psoriasis. N Engl J Med. 2018;379(14):1313-21.

16. Armstrong A, Gooderham M, Warren R, et al. Efficacy and safety of deucravacitinib, an oral, selective tyrosine kinase 2 (TYK2) inhibitor, compared with placebo and apremilast in moderate to severe plaque psoriasis: results from the POETYK PSO-1 study [abstract]. In: Presented at: the annual meeting of the American Academy of Dermatology; April 23-25, 2021

17. Girgis IG, Chimalakonda A, Kundu S, et al. Exposure-efficacy response modelling of BMS-986165, an oral, selective tyrosine kinase 2 (TYK2) inhibitor, in a phase 2 study in psoriasis [poster P066]. Presented at: the triennial meeting of the Skin Inflammation \& Psoriasis International Network; April 25-27, 2019; Paris, France

18. Klünder B, Mittapalli RK, Mohamed MF, et al. Population pharmacokinetics of upadacitinib using the immediate-release and extended-release formulations in healthy subjects and subjects with rheumatoid arthritis: analyses of phase I-III clinical trials. Clin Pharmacokinet. 2019;58(8):1045-58.

19. Xie R, Deng C, Wang Q, et al. Population pharmacokinetics of tofacitinib in patients with psoriatic arthritis. Int J Clin Pharmacol Ther. 2019;57(9): 464-73.

20. Zhang X, Chua L, Ernest C 2nd, et al. Dose/exposure-response modeling to support dosing recommendation for phase III development of baricitinib in patients with rheumatoid arthritis. CPT Pharmacomet Syst Pharmacol. 2017;6(12):804-13.

21. Dowty ME, Lin TH, Jesson MI, et al. Janus kinase inhibitors for the treatment of rheumatoid arthritis demonstrate similar profiles of in vitro cytokine receptor inhibition. Pharmacol Res Perspect. 2019;7(6):e00537.

22. Schwartz DM, Kanno Y, Villarino A, et al. JAK inhibition as a therapeutic strategy for immune and inflammatory diseases. Nat Rev Drug Discov. 2017;16(12):843-62.

23. Gordon K, Papp K, Gooderham M, et al. BMS986165, an oral, selective tyrosine kinase 2 (TYK2) 
inhibitor: evaluation of changes in laboratory parameters in response to treatment in a Phase 2 trial in psoriasis [poster]. Presented at: the American Academy of Dermatology Annual Meeting; March 20-24, 2020; Denver, CO

24. Mease PJ, Deodhar A, van der Heijde D, et al. Efficacy and safety of deucravacitinib (BMS-986165), an oral, selective tyrosine kinase 2 inhibitor, in patients with active psoriatic arthritis: results from a phase 2, randomized, double-blind, placebo-controlled trial [poster L03]. Presented at: the annual scientific meeting of the American College of Rheumatology; November 5-9, 2020; virtual meeting
25. McInnes IB, Byers NL, Higgs RE, et al. Comparison of baricitinib, upadacitinib, and tofacitinib mediated regulation of cytokine signaling in human leukocyte subpopulations. Arthritis Res Ther. 2019;21(1):183.

26. Mohamed MF, Beck D, Camp HS, Othman AA. Preferential inhibition of JAK1 relative to JAK3 by upadacitinib: exposure-response analyses of ex vivo data from 2 phase 1 clinical trials and comparison to tofacitinib. J Clin Pharmacol. 2020;60(2):188-97.

27. Dowty ME, Jesson MI, Ghosh S, et al. Preclinical to clinical translation of tofacitinib, a Janus kinase inhibitor, in rheumatoid arthritis. J Pharmacol Exp Ther. 2014;348(1):165-73. 